\title{
COMUNICADO DE ASONEUMOCITO SOBRE EL ASBESTO EN COLOMBIA
}

\section{STATEMENT OF ASONEUMOCITO ABOUT ASBESTOS IN COLOMBIA}

José Gabriel Bustillo Pereira, MD. ${ }^{(1)}$

La palabra asbesto o amianto, hace relación a un grupo de silicatos fibrosos (amfiboles y serpentinas) que debido a sus propiedades de durabilidad y gran resistencia al calor y a la tracción, son ampliamente utilizados, entre otras, en las industrias de la construcción, elaboración de frenos para automóviles y cableado eléctrico, las cuales consumen las cerca de 2 millones de toneladas que algunos de los países productores aún continúan lanzando anualmente al mercado.

Desde el punto de vista de la Medicina ocupacional y la Salud pública, se dejan escuchar, cada vez con mayor frecuencia, voces que alertan sobre los riesgos que corren, no solo los manipuladores directos de este material, sino también sus familiares y algunos sectores de la población en general, quienes de manera directa o indirecta se ven expuestos al contacto con el asbesto, por tiempos y cantidades que varían de acuerdo con los datos revisados.

La situación no es nueva. Ya desde 1955, Richard Doll, el mismo influyente y encumbrado epidemiólogo inglés que junto con Bradford Hill llamaran la atención sobre la relación entre tabaquismo y cáncer pulmonar, dejó sentado en un histórico estudio, la también innegable relación existente entre cáncer pulmonar y mortalidad en los trabajadores de asbesto (1 (Doll R, Mortality from lung cancer in asbestos workers. Br J Indust Med 1955; 12: 81-6. Reimpreso en el Br J Indust Med. 50(6): 48590, junio 1993). De allí en adelante son numerosos los trabajos que con metodología científica incuestionable llegan a conclusiones similares; lo anterior sin agregar los pronunciamientos de la Organización Inernacional de Trabajo y la Organización Mundial de la Salud acerca de las propiedades cancerígenas de todas las formas de asbesto, consideraciones que hasta ahora han conducido a unos 52 países en el mundo a la revisión de sus legislaciones y a la posterior suspensión de la explotación, uso y comercialización de este material, contándose todavía algunas naciones en vías de desarrollo que bajo una inexplicable tolerancia, hablan de "formas seguras en el manejo del crisotilo"

No desconocemos el impacto laboral que supondría la potencial reubicación de los muchos trabajadores del área, situación que no obstante, a largo plazo contaría con menores
(1)Presidente Asociación Colombiana de Neumología y Cirugía de Tórax. Correspondencia: José Gabriel Bustillo Pereira, correo electrónico: jose bustillo@juanncorpas.edu.co Recibido: 02/07/15. Aceptado: 04/08/15. 
repercusiones sociales que las que pueden esperarse del hecho de que los intereses de la industria primen sobre el de la salud de los ciudadanos, tal como ha venido sucediendo con la industria del tabaco. Por todo lo anterior, como entidad académica rectora de la neumología en el país, expresamos nuestra solidaridad con la población afectada, y en tono muy respetuoso pero enérgico, sugerimos al gobierno nacional y a la empresa privada, que en lugar de insistir infructuosamente en la ausencia de una relación unicausal entre las fibras de asbesto y la generación de cáncer, nos acojamos más bien a la poderosa y creciente evidencia de asociación entre la exposición al asbesto y el cáncer pulmonar, la cual permite formular sobradas recomendaciones para abolir la explotación y el uso del mineral en cuestión.

\section{Lecturas recomendadas}

- Greenberg MB. A Study of Lung Cancer Mortality in Asbestos Workers: Doll, 1955.American Journal Of Industrial Medicine 1999, 36:331 \pm 347
- Prazakova S, Thomas PS, Sandrini A, Yates DH. Asbestos and the lung in the 21 st century: an update. The Clinical Respiratory Journal 20014; 1-10

- Collegium Ramazzini. Letter to the Editor. Comments on the causation of malignant mesothelioma: rebutting the false concept that recent exposures to asbestos do not contribute to causation of mesothelioma J Occup Health 2016; 58: 228-229

- $\quad$ Egilman D, Bird T, Lee C. Dust diseases and the legacy of corporate manipulation of science and law. International journal of Occupational and Environmental Health 2014; 20,2: 115-126

- Gilham C, Christine Rake C, Garry Burdett G, Nicholson AG, Davison L, Franchini A, Carpenter J, Hodgson J, Darnton A, Peto J. Pleural mesothelioma and lung cancer risks in relation to occupational history and asbestos lung burden . Occup Environ Med 2016;73:290-299

- $\quad$ Philips CV, Goodman KJ. The missed lessons of Sir Austin Bradford Hill. Epidemiologic Perspectives \& Innovations 2004, 1:3

- Banegas JR, Fernando Rodríguez F, Calero J. Popper y el problema de la inducción en epidemiología. Rev Esp Salud Pública 2000; 74;327-339 N. 4 